\title{
RADIO MEASUREMENT OF THE TIME DELAY IN $0218+357$
}

\author{
E.A. CORBETT, I.W.A. BROWNE AND P.N. WILKINSON
}

University of Manchester, Nuffield Radio Astronomy Laboratories

Jodrell Bank, Macclesfield, Cheshire, SK11 9DL, U.K.

AND

A.R. PATNAIK

Max-Planck-Institut für Radioastronomia

Auf dem Hügel 69, D-53121 Bonn, Germany

\begin{abstract}
A time delay of $12 \pm 3$ days has been measured for the lens system $0218+357$ using VLA $15 \mathrm{GHz}$ polarization observations.
\end{abstract}

\section{Introduction}

The potential for using a measurement of the time-delay in a gravitational lens system to give a value for the Hubble constant has long been appreciated (Refsdal 1964). However, only a few systems suitable for such a determination have been found. The $0218+357$ system (Patnaik et al. 1993), found in JVAS (The Jodrell/VLA Astrometric Survey - see Patnaik et al. 1992), is one of the most promising. It consists of 2 images of a strong flat spectrum radio core plus a low brightness ring of emission (Figure 1). The 2 core images are compact, have high radio polarizations ( 10\%) and show evidence for radio variability. Both the ring and VLBI images of the compact core images (Patnaik et al. 1995) give useful constraints on the mass distribution of the lensing galaxy. The lensing galaxy redshift has been measured to be 0.6847 from an [OII] $3727 \AA$ emission line and optical absorption lines (Browne et al, 1993). It has been confirmed by the detection of $21 \mathrm{~cm}$ neutral hydrogen absorption (Carilli et al. 1993) and recently by $\mathrm{CO}$ and other molecular absorption lines (Wicklind \& Combes 1995). Lawrence et al. (in these proceedings) report the detection of a broad emission line and associated absorption from which they propose a redshift 
of 0.96 for the lensed object. In this contribution we report the successful determination of a time delay in $0218+357$.

\section{VLA Observations}

Observations were made with the VLA ${ }^{1}$ in its A-configuration at frequencies of $15 \mathrm{GHz}$ and $8.4 \mathrm{GHz}$ on 25 irregularly spaced occasions during 3 -months in $1992 / 3$. The resolution of the VLA at these 2 frequencies is 0.12 arcsec and 0.2 arcsec respectively, adequate to separate the compact images which are 0.335 arcsec apart. We set out to monitor the total flux densities, the polarized flux densities and polarization position angles of the compact images. As well as $0218+357$, three calibration sources and a "control source" were observed during the 45 minutes allocated to each epoch.

\section{Data Analysis}

Owing to the short duration of the observations at each epoch, some nonstandard calibration methods were necessary. For flux density calibration we assumed that the VLA primary calibrator 3C 84 had constant flux density over the period of observation. When all epochs had been calibrated, the validity this assumption was checked by seeing if the flux densities derived in this way for the CSS sources 3C48, 3C119 and 3C147 were constant with time. This proved to be the case. The antenna polarization residual terms were also derived from the $3 \mathrm{C} 84$ observations on the assumption that $3 \mathrm{C} 84$ is unpolarized - something supported by the long-term monitoring program of Aller \& Aller (personal communication). The zero of polarization position angles was set arbitrarily with respect to that of the position angle of $3 \mathrm{C} 119$.

The target, the calibration sources and the control source (3C119) were all mapped using an AIPS procedure involving 2 cycles of phase selfcalibration. Stokes I, Q and U flux densities for all but the target source were derived using the AIPS task JMFIT. In the case of $0218+357$, fitting in the image plane proved not to be a satisfactory method to derive the core image flux densities because the core images are blended with the extended ring emission. Instead we exploited the fact that the ring emission is almost completely resolved at baselines $\geq 400 \mathrm{k} \lambda$. We excluded all baselines shorter than $400 \mathrm{k} \lambda$ from the self-calibrated data-set and used the task UVFIT to model-fit 2 point components to the visibility data. The component separation and position angle were fixed to the values found from high resolution MERLIN and VLBI maps. In this way I, $Q$ and U flux densities

\footnotetext{
${ }^{1}$ The VLA is operated by Associated Universities, Inc., under a cooperative agreement with the National Science Foundation.
} 
were obtained for the two images, uncontaminated by the low brightness emission.

\section{Results}

The peak-to-peak errors in total flux density are $\sim 7 \%$ at $15 \mathrm{GHz}$ and $\sim 3 \%$ at $8.4 \mathrm{GHz}$ due to uncertainties in calibration. These uncertainties masked any clear signal that could be used to determine a time delay, but it was evident that the ratio of the flux densities of the $0218+357$ images varied, indicating real variability on a time scale comparable to, or shorter than, the time delay. Small residual errors in the polarization position angle calibration compromise the usefulness of this parameter for time delay measurements. On the other hand the percentage polarizations of the two images show clear variations. The $15 \mathrm{GHz}$ polarization light curves are shown in Figure 2. The $8.4 \mathrm{GHz}$ results show similar trends with the percentage polarizations of both the $\mathrm{A}$ and $\mathrm{B}$ images increasing with time. The formal errors on the measured percentage polarizations are $\leq 0.2 \%$. Moreover, systematic flux density calibration errors cancel. Hence percentage polarization is a much more robust parameter to use in a time delay determination. In Figure 3 we show the $15 \mathrm{GHz}$ percentage polarization light curve for the control source $3 \mathrm{C} 119$. In contrast to the steady increase in the percentage polarization seen in $0218+357,3 \mathrm{C} 119$ has constant polarization. We note that the percentage polarization of the strongest image (A) of $0218+357$ shows significant changes on time scales as short as 2 or 3 days - something that should enable the time delay to be measured to an accuracy of $\sim 1$ day if similar variations can be detected in $\mathrm{B}$.

The polarization properties of the images of a point object should be identical. There are, however, complications. The first is that, if the object being imaged has internal polarization substructure, different magnification gradients across the images could give rise to each image having different integrated polarizations. In the case of $0218+357$ we know that the $15 \mathrm{GHz}$ substructure has a size of only $\sim 1$ mas (Patnaik et al. 1995) - a scale over which the models indicate that the magnifications are essentially constant. Therefore this is not a problem in the present case.

An additional complication is that we view the images through the ISM lensing galaxy and the two ray paths suffer different degrees of Faraday rotation, and even Faraday depolarization. Both effects occur in the $0218+357$ system; the rotation measures of the two images differ by 814 radian $\mathrm{m}^{-2}$ and, at frequencies of $5 \mathrm{GHz}$ and lower, the percentage polarization of the $\mathrm{A}$ image is much less than that of the B image, something that we attribute to depolarization caused by the Faraday depth changing across the face of the A-image. In the case of the Faraday rotation it is possible to determine 
the rotation measure difference and correct for it on the assumption that a $\lambda^{2}$ law is obeyed. The way to avoid the effects of depolarization is to observe at high frequencies. We think $15 \mathrm{GHz}$ is high enough to be free from this problem and hence we base most of our conclusions on observations at this frequency. We also note that the A-image is the most depolarized. It is this image that has the higher average percentage polarization during the period of our observations. Thus any small residual depolarization in A will result in the separation in the light curves for the two images (Figure 2 ) being underestimated. This would also lead to our measured time delay being an underestimate of the true time delay.

\section{Time Delay Analysis}

We slide the polarization light curve for one image relative to the other and minimize the difference to give the delay. We have done this visually and, more formally, by linearly interpolating between the measurements in one light curve and adjusting the time delay to minimize $\chi^{2}$ (normalized for the decreasing number of epochs compared for increasing delays) between the two time series. The plot of the normalized $\chi^{2}$ against delay is shown in Figure 4. There is a distinct minimum at a delay of 12 days. The formal result is that the delay is 12 days with a $1 \sigma$ error of \pm 3 days.

\section{Conclusions}

- (1) We have determined the time delay in the gravitationally lensed system $0218+357$ from the variation of the radio percentage polarization of the images with time. The delay is $12 \pm 3$ days.

- (2) Given the redshift of the lensed object measured by Lawrence et al. (this conference), the value for the Hubble constant obtained is $\sim 60 \mathrm{~km} \mathrm{~s}^{-1} \mathrm{Mpc}^{-1}$ (Nair, this conference).

- (3) With more frequent and regular sampling of the polarization light curve we can greatly improve the accuracy of our measurement of time delay in the future.

\section{References}

Browne, I.W.A., et al., 1993, MNRAS, 263, L32

Carilli, C.L., et al., 1993, ApJL, 412, L59

Patnaik, A.R., et al., 1992, MNRAS, 254, 655

Patnaik, A.R., et al., 1993, MNRAS, 261, 435

Patnaik, A.R., Porcas, R.W. \& Browne, I.W.A., 1995, MNRAS, 274, L5

Refsdal, S., 1964, MNRAS, 128, 307

Wiklind, T. \& Combes, F., 1995, A\&A, 299, 382 
TIME DELAY IN 0218+357

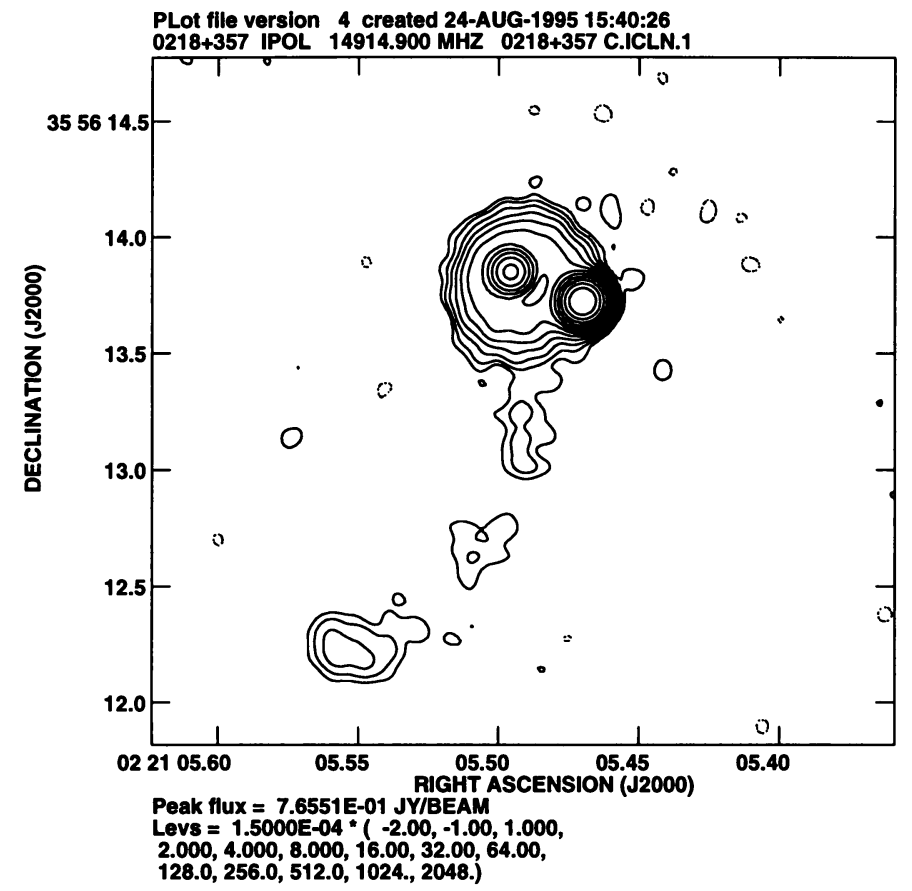

Figure 1. VLA $2 \mathrm{~cm}$ map of $0218+357$ showing the two core images, the ring and a weak jet.

B $0218+357 \%$ Polarisation, IF Average, $15 \mathrm{GHz}$

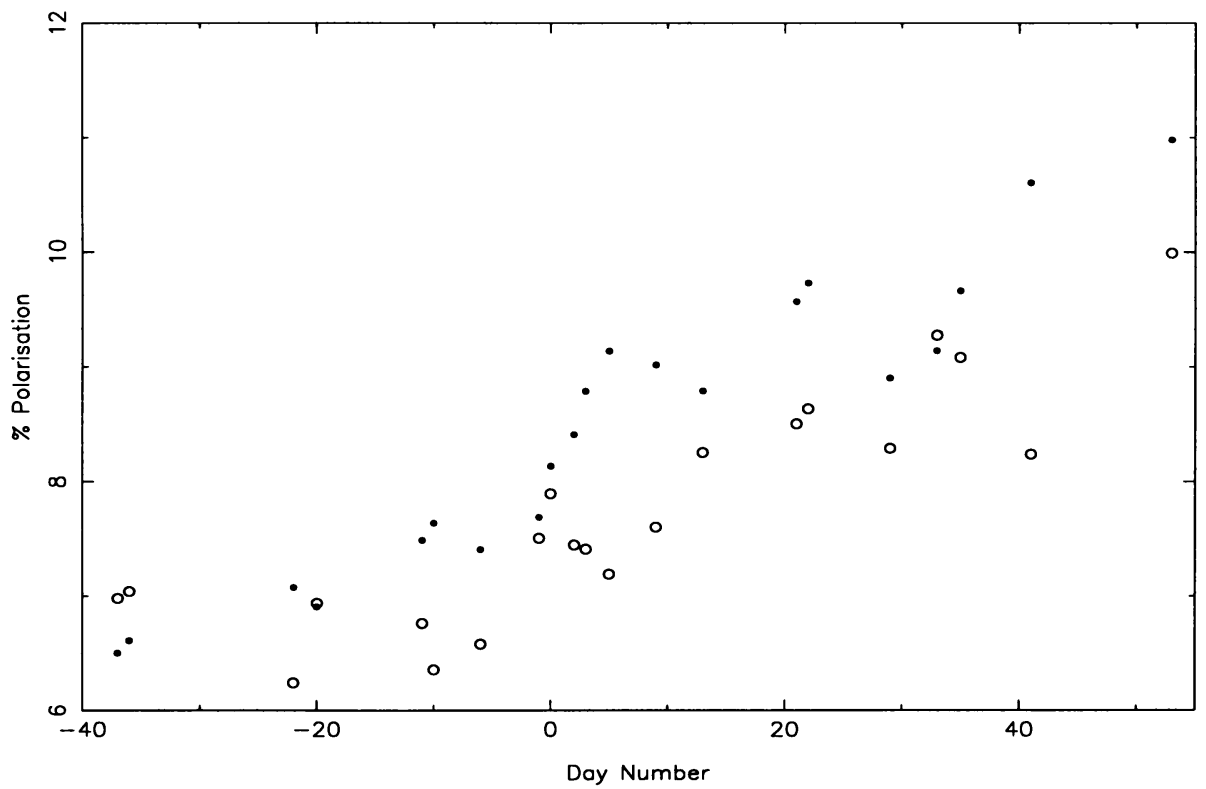

Figure 2. Percentage polarization of the A-image (filled circles) and B-image (open circles) as a function of time. 
3C119 Data,\% Polarisation, IF 1 and $2,15 \mathrm{GHz}$

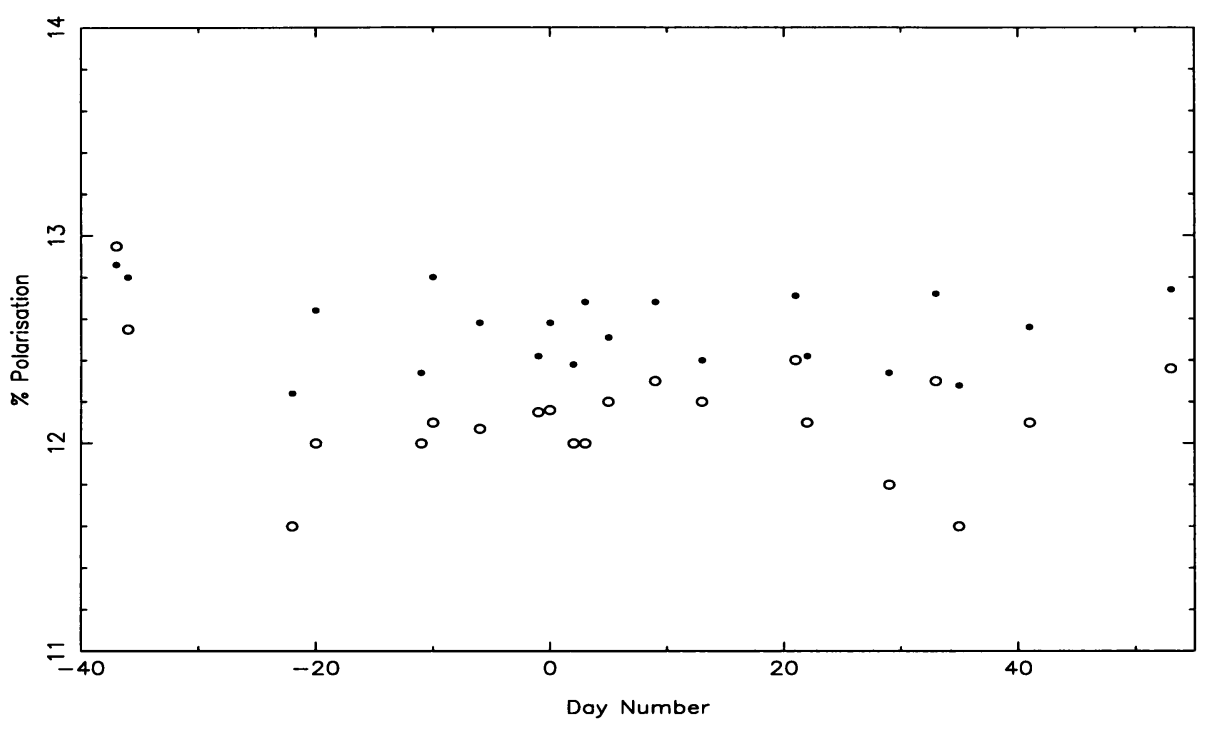

Figure 3. The percentage polarization as a function of time for the control source 3C119. The two VLA IFs are distinguished by different symbols.

\% Polarisation, Chi-Squared Test, Averaged IFs, $15 \mathrm{GHz}$

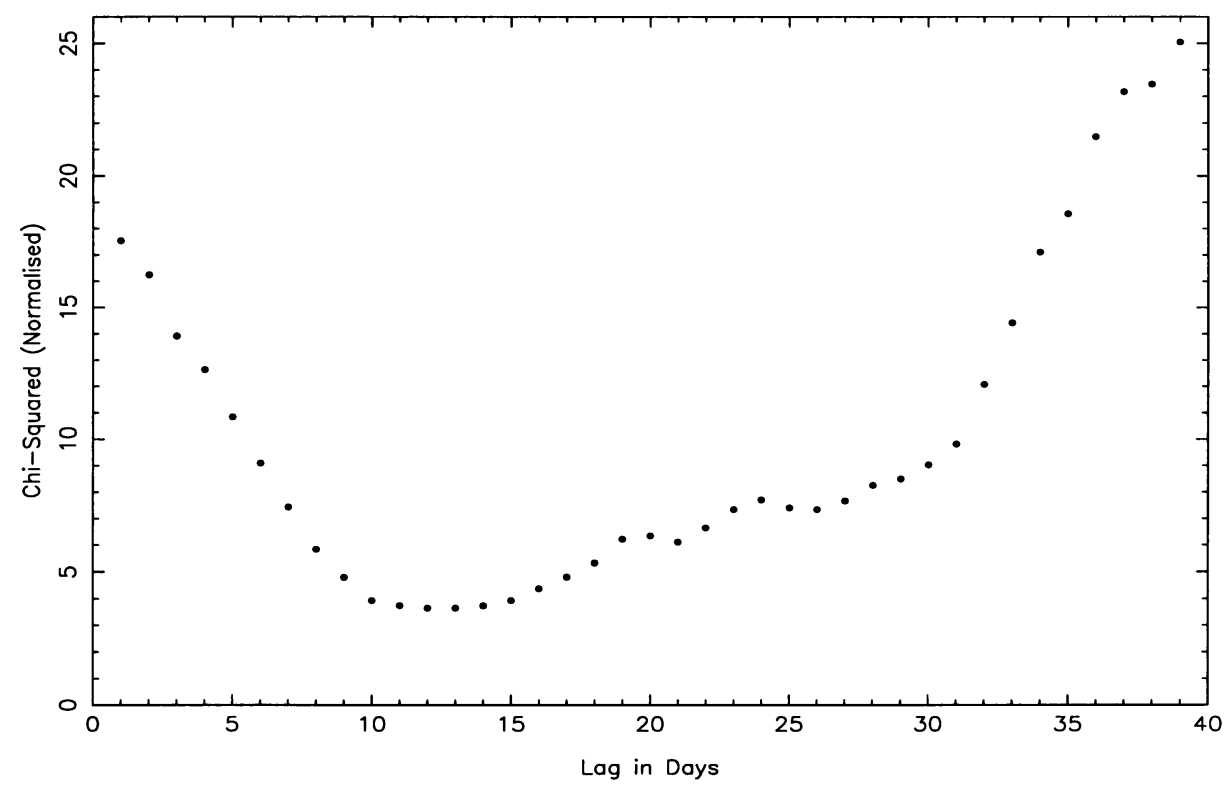

Figure 4. The Normalized $\chi^{2}$ as a function of time lag between the A and B polarization light curves. 